\title{
Cost Analysis for Biodiesel Production from Waste Cooking Oil Plant in Egypt
}

\author{
Abd Allah S. A. El-Gharabawy \\ The Egyptian Ethylene \& Derivatives Company - ETHYDCO, Alexandria, Egypt \\ ab_pet_88@hotmail.com
}

Received: 26.11.2017 Accepted:30.12.2017

\begin{abstract}
The depletion of fossil fuel forces the world to look for alternative ways for energy production. The most common way is the production of biodiesel from waste cooking oil. This paper presents a detailed cost analysis for a biodiesel plant with capacity 100,000 ton/year. The cost analysis basis depends on the optimum conditions obtained from the experimental work that achieves biodiesel conversion of $\approx 99 \%$. The study concludes that the project will achieve a good income that around four million US \$ every year and the total net income during the life cycle of project is 72.5 million US \$. The production cost of one liter biodiesel from used cooking oil is 0.515 \$ while the global price of petro-diesel is 0.678 \$ liter and global biodiesel current price is 1 \$/liter.
\end{abstract}

Keywords: Biodiesel, Cost analysis, Waste cooking oil

\section{Introduction}

Biodiesel is a fatty acid alkyl ester produced from biological sources such as vegetable oil, animal fats wastes and algae. Production of biodiesel from non-edible vegetable oil hits a lot of benefits [1,2]. Economically, biodiesel is more profitable than petro-diesel as biodiesel plants does not cost more money like refining plants, in addition low cost of biodiesel feedstock rather than expensive crude oil the feedstock of petro-diesel. Production of biodiesel is a simple process that does need neither a license nor a complicated technology. Environmentally, biodiesel is better as it does not contain sulfur so sulfur emissions is eliminated. It is renewable as biodiesel is produced from renewable resources such as used cooking oil or biological organisms while petrodiesel made from finite sources. The aim of cost analysis is to estimate the budget required to build up and operate a project and predict the profitability of project. The study in this work applied on a plant with capacity 100,000-ton biodiesel. The operating assumption basis was 7.54:1 Methanol to Oil ratio, $0.875 \mathrm{wt} \% \mathrm{KOH}$, a temperature of $52.7^{\circ} \mathrm{C}$, a time of $1.17 \mathrm{~h}$ and agitation speed of $266 \mathrm{rpm}$. The capital expenditure (CAPEX) of the project is the cost needed to build up a plant. CAPEX is divided into purchased equipment cost (PEC) and other CAPEX. The purchased equipment (PEC) is the cost needed for biodiesel production equipment while other CAPEX is the costs corresponding to plant build-up such as equipment installation, instrumentation, piping, electrical, and construction. The operating cost (OPEX) is the expenses needed to operate the plant every year. The OPEX divided into fixed cost and variable cost. Fixed cost is a yearly-fixed expenses such as taxes, insurance, and labor salaries. Variable cost is a yearly non-fixed expense such as raw materials, utilities and chemicals.

\section{Cost Analysis Basis}

The structure of the financial model on MICROSOFT EXCEL PROGRAM is a series of sheets connected with each other by mathematical equations. The main excel sheets are basics of calculations sheet, total capital investment cost sheet, annual operating cost sheet, annual revenues sheet, products, by-products, utility and feedstocks Prices sheet and income statement sheet. The model also contains an auxiliary sheet, which is the loan drawdown, and installments sheet that is used in the case of the project finance technique is divided into equity and debt.

\subsection{Technical Assumptions}

Construction period of biodiesel project will be two years from basic engineering till start-up including all issues related to equipment purchased for the project, financial obligations ,construction phase , pre-commissioning phase , commissioning and start-up phase .The construction period will begin at June 2017 till June 2019 the start-up phase . Operating capacity which is $100 \mathrm{KTA}$ of biodiesel will be 80 KTA in 2019 and 90 KTA in 2020 as the plant cannot operate with full capacity during its first and second year, after second year of project the plant will operate with full capacity for the whole project life cycle. Project life cycle will be 20 years with 7200 operating hours per year. 


\subsection{Economic Assumptions}

Inflation is defined as an increase in a currency supply relative to the number of people using it. As a result of this supply inflation the general price level of goods and services in an economy over a period of time rise. When the price level rises, each unit of currency buys fewer goods and services [3].

Inflation rate for Opex, material prices, catalyst, chemicals, and feedstock is considered to be $3 \%$ of its price while labor inflation rate will be $8 \%$.

The project will be executed by $49 \%$ equity and 51\% debit with loan interest $9 \%$ which is high value as in most projects the loan interest is $6 \%$. The debt repayment period or payback period is 8 years as shown in table 1 .

\subsection{Operating Assumptions}

The project is designed to process 101,000 ton of used cooking and 26,800 ton of methanol to produce 100,000 ton of biodiesel. The amount of cooling water and process water used during production will be at most 1 million cubic meters per year and steam for heat exchanging will be around 300,000 ton per year. Chemical cost will be at most 3 million per year and area of land is 42,000 square meter that equal 10 feddan as shown on table 2 .

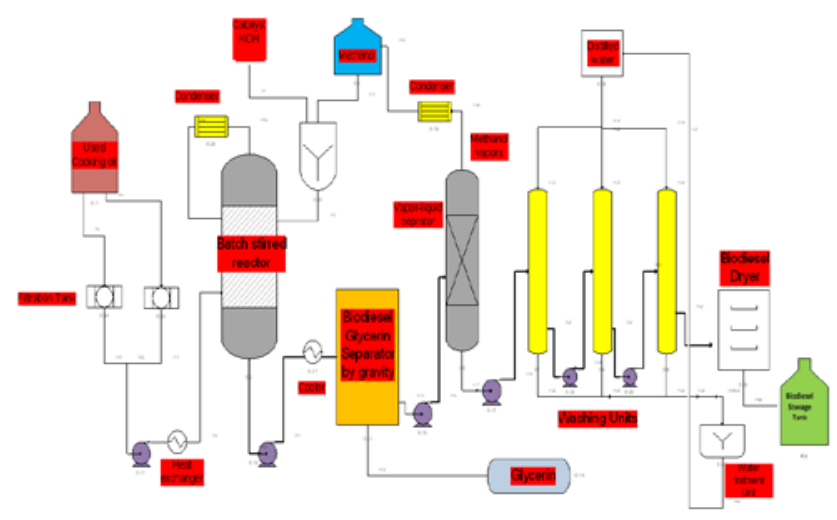

Fig 1: Alkali-catalyzed transesterification of WCO-process

Table 1: Biodiesel Project Technical \& Economic Assumptions

\begin{tabular}{|l|l|}
\hline Technical Assumption & Value \\
\hline Construction Period (year) & 2 \\
\hline Project Life Cycle (year) & 20 \\
\hline Startup Date & $01 / 06 / 2019$ \\
\hline working hours per year & 7,200 \\
\hline Operating Capacity & Value \\
\hline Year 1 & $80 \%$ \\
\hline Year 2 & $90 \%$ \\
\hline Year 3+ & $100 \%$ \\
\hline Economic Assumptions & Value \\
\hline Inflation Rate & $3 \%$ \\
\hline Labour Inflation Rate & $8 \%$ \\
\hline Equity & $49 \%$ \\
\hline
\end{tabular}

\begin{tabular}{|l|l|}
\hline Debt & $51 \%$ \\
\hline Loan Interest & $9 \%$ \\
\hline Debt Repayment Period (years) & 7 \\
\hline
\end{tabular}

Table 2: Biodiesel Operating Assumptions

\begin{tabular}{|l|l|}
\hline Products & Value \\
\hline Biodiesel & $100 \mathrm{KTA}$ \\
\hline Feedstock & $26.8 \mathrm{KTA}$ \\
\hline Methanol & $0.875 \mathrm{KTA}$ \\
\hline Potassium hydroxide & $101 \mathrm{KTA}$ \\
\hline Used Cooking Oil Wrocess ater & $\begin{array}{l}1,000,000 \\
\mathrm{~m}^{3} / \text { Year }\end{array}$ \\
\hline Utilities & $\begin{array}{l}300,000 \\
\text { ton/year }\end{array}$ \\
\hline $\begin{array}{l}\text { Cooling Consumption without recirculation } \\
\text { Steam Consumptions }\end{array}$ & $15,000(\mathrm{MWH})$ \\
\hline Electricity Consumption & $\begin{array}{l}3 \\
\text { MMUSD/Year }\end{array}$ \\
\hline Chemicals and Catalyst Cost & $42,000 \mathrm{~m}^{2}$ \\
\hline Land Requirement & 150 \\
\hline Labor Requirement (number) &
\end{tabular}

Table 3: Biodiesel labors Assumptions

\begin{tabular}{|l|l|}
\hline No. of labors & 150 \\
\hline Average salary (\$) & 1800 \\
\hline Total salaries per month (\$) & 270000 \\
\hline Total salaries per year (Million \$) & 3.24 \\
\hline
\end{tabular}

\section{Cost Analysis Design}

This study is concerning with evaluation the economic costs of transforming crude used cooking oil into a methyl ester product via commercial plant scale with capacity 100,000 ton per year.

\subsection{Capital expenditure}

Capital costs are the costs that expensed one time at the beginning of project or expenses need to build up a project and prepare it to the operation phase. It is fixed cost such as land, equipment, buildings, instrumentation, piping, etc.

\subsubsection{Purchased Equipment Cost (PEC)}

The purchased equipment needed for biodiesel production is as follow.

\section{A. Tanks}

Carbon steel tanks were sized for storage of used cooking oil, methanol, potassium hydroxide, glycerol and produced 
biodiesel. Settling tanks and gravity separators are used to separate the produced diesel and glycerol depend on higher density of glycerol. Tanks with capacity 10,000 cubic meters will be sufficient to store all this amount of reactant and product while tank with capacity 1000 will be used to store potassium hydroxide and material of tank will be carbon steel as no utilization of acid or corrosive materials.

\section{B. Batched stirred reactor}

Transesterification can be carried out discontinuously (batch). Batch stirred tank reactor is a very simple type of reactor. It consists of a reactor, mixer and a propeller. Its dimension $2200 * 2200 * 4500$ which can process about 15 cubic meter of used cooking oil per batch with time 3 hours which equal 120 cubic meter per day for one reactor .

Volume of used cooking $=120 * 300$ (working day) oil processed annually by

one reactor

$$
=36000 \mathrm{~m} 3
$$

Number of reactors required $=3$

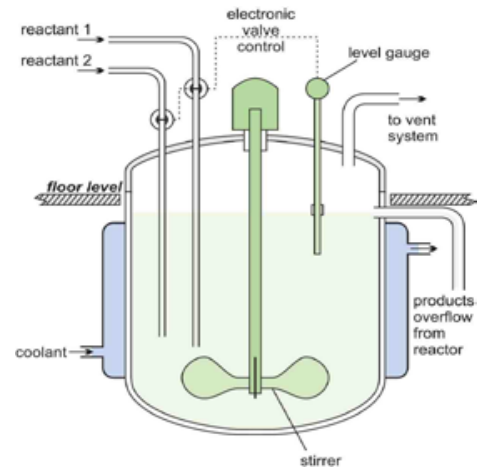

Fig 2 :Batched stirred reactor

\section{Vapor-liquid separator}

Vapor-liquid separator is used to separate methanol traces in final product of biodiesel depending on boiling of methanol at $70^{`} \mathrm{C}$ while biodiesel remaining as liquid.

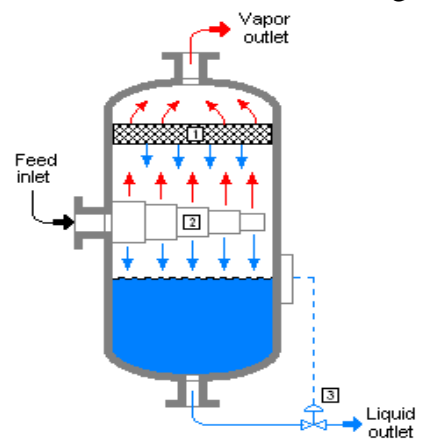

Fig 3 :Vapor-liquid separator

\section{Mixer}

Mixer will be used to mix methanol with potassium hydroxide. The size of mixer will be purchased based on processing of $12 \mathrm{~m} 3 / \mathrm{hr}$ of methanol needed for one batch for three reactors.

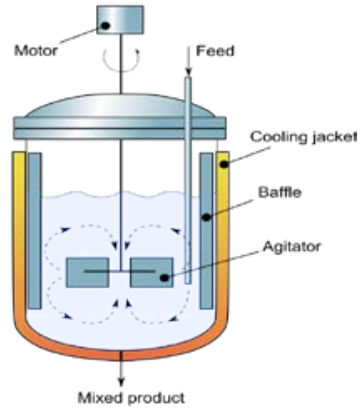

Fig 4 :Mixer

\section{E. Filters}

Filters will be used to remove the insoluble impurities in the feedstock which may consist of sand, dirt, and small particles of bones. Feedstocks should always be filtered prior to entering the processing system as it may affect the internal part of equipments.

\section{F. Heat Exchangers}

Heat exchanger is a piece of equipment used to achieve maximum benefit of heat recovery. There will be two heat exchangers used one for oil feedstock before inlet of main reactor and the other for produced mixture of diesel and glycerol before settling tank.

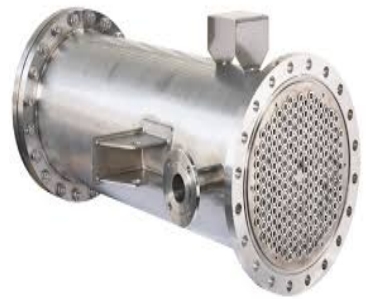

Fig5: Heat Exchanger

\section{G. Condenser}

Condenser will be used to condense unreacted vapors of methanol and return back to process so as to achieve the maximum benefit of process. There will be two condensers the first one dedicated to main reactor and the other dedicated to methanol separator.

\subsection{Other CAPEX}

Unit cost estimates will result in accurate estimates for fixed capital investments if accurate records have been kept from previous cost experience. This method also requires detailed estimates of purchased prices obtained from either indexcorrected cost records and published data or quotations. Pipe, steel, concrete, insulation, instrumentation, and other such costs are obtained by take-offs from the drawings and applying unit costs to the material and labor needs. Equipment installation labor costs are specified as a fraction of the delivered equipment cost. A factor for the contractor's fee, construction expenses, and contingency is estimated 
from previously completed projects. Percentage of delivered purchased equipment cost (PEC) requires careful determination of equipment cost. Other items that can be estimated as percentages or purchased equipment cost are as follows:

- Equipment installation.

- Instrumentation and controls.

- Piping, installed.

- Electrical, installed.

- Buildings.

Costs for engineering and supervision, construction, contractor's fees, and contingency can be based on average percentages of the physical plant cost (PPC) or PPC plus erection costs (PPC \& EC). This method is commonly used for preliminary estimates.

Table 4: Total capital cost

\begin{tabular}{|c|c|c|}
\hline Item & Percentage & Price (\$) \\
\hline $\begin{array}{c}\text { Equipment } \\
\text { Installation }\end{array}$ & $35 \%$ & 455000 \\
\hline Instrumentation & $20 \%$ & 260000 \\
\hline Piping & $66 \%$ & 858000 \\
\hline Electrical & $11 \%$ & 143000 \\
\hline Building & $45 \%$ & 585000 \\
\hline $\begin{array}{c}\text { Transportation } \\
\text { with contingency } \\
\text { reserves }\end{array}$ & $15 \%$ & 195000 \\
\hline PEC & $25 \%$ & $3,796,000$ \\
\hline Total CAPEX & & $1,300,000$ \\
\hline
\end{tabular}

Table 5: Purchase Equipment Cost

\begin{tabular}{|c|c|c|c|c|}
\hline Equipment & No. & $\begin{array}{c}\text { Price US } \\
\text { (\$) }\end{array}$ & $\begin{array}{l}\text { Total } \\
\text { price }\end{array}$ & Reference \\
\hline $\begin{array}{l}\text { Carbon } \\
\text { steel Tank } \\
\text { with } \\
\text { capacity } \\
10000 \mathrm{~m}^{3}\end{array}$ & 10 & 30,000 & 300,000 & $\begin{array}{l}\frac{\underline{\text { http://www.alibaba.co }}}{\mathrm{m} / \text { product- }} \\
\frac{\underline{\text { detail/Carbon-Steel- }}}{\text { Storage- }} \\
\underline{\underline{\text { Tank_737506719.html }}} \\
\underline{\text { ?s=p }}\end{array}$ \\
\hline $\begin{array}{c}\text { Tank with } \\
\text { capacity } \\
1000 \mathrm{~m}^{3} \\
\end{array}$ & 2 & 20,000 & 40000 & \\
\hline Condenser & 3 & 100,000 & 300000 & \\
\hline $\begin{array}{c}\text { Heat } \\
\text { exchanger }\end{array}$ & 4 & 15,000 & 60000 & $\begin{array}{l}\frac{\text { http://www.alibaba.co }}{\text { m/product-detail/heat- }} \\
\frac{\text { exchanger_601591672 }}{\text { 92.html?s=p }}\end{array}$ \\
\hline $\begin{array}{c}\text { Carbon } \\
\text { steel } \\
\text { Vessels }\end{array}$ & 3 & 30,000 & 90000 & $\begin{array}{l}\frac{\text { http://www.alibaba.co }}{\mathrm{m} / \text { product-detail/10- }} \\
\frac{\text { cubic-meters-reaction- }}{\text { vessel- }} \\
\frac{\text { manufacturer_149959 }}{\text { 2917.html }}\end{array}$ \\
\hline $\begin{array}{c}\text { Pumps } \\
\text { with flow } \\
\text { rate } 10 \mathrm{~m}^{3}\end{array}$ & 8 & 20,000 & 160000 & \\
\hline Filters & 4 & 15,000 & 60000 & $\begin{array}{c}\frac{\text { http://www.alibaba.co }}{\text { m/product- }} \\
\frac{\text { detail/supply-metal- }}{\text { fuel-solid-liquid- }} \\
\text { gas_1633192375.html }\end{array}$ \\
\hline $\begin{array}{c}\text { Batched } \\
\text { stirred } \\
\text { reactor }\end{array}$ & 3 & 50,000 & 150000 & $\begin{array}{l}\frac{\text { http://www.alibaba.co }}{\mathrm{m} / \text { product-detail/- }} \\
\frac{\underline{\text { strong-batch-strong- }}}{\underline{\text { strong- }}} \\
\frac{\text { stirred 864249397.ht }}{\underline{\mathrm{ml}}}\end{array}$ \\
\hline Mixer & 2 & 20000 & 40000 & $\begin{array}{c}\frac{\text { http://www.alibaba.co }}{\underline{\text { m/product- }}} \\
\frac{\text { detail/chemical- }}{\text { mixing-reactors- }} \\
\underline{\text { stainless-steel- }} \\
\underline{\text { jacketed_6023627520 }} \\
\underline{1 . h t m l ? s=p}\end{array}$ \\
\hline $\begin{array}{l}\text { Vapour- } \\
\text { liquid } \\
\text { separator }\end{array}$ & 2 & 50,000 & 100000 & $\begin{array}{c}\frac{\text { http://www.alibaba.co }}{\text { m/product- }} \\
\underline{\text { detail/STLB60- }} \\
\begin{array}{c}\text { Centrifugal-Gold- } \\
\text { Separator_105224426 }\end{array} \\
\underline{\text { 4.html?s=p }}\end{array}$ \\
\hline Total & & & $1,300,000$ & \\
\hline
\end{tabular}




\subsection{Operating Costs (OPEX)}

Operating costs are the expenses that related to the operation of plant. Operating costs divided into fixed and variable costs. Fixed costs are those incurred whether the plant is in production or not and are at a constant level regardless of the output produced such as salaries or rents being paid per month. Variable costs vary at a rate directly related to the production of the plant. They are incurred only when the plant is in operation, although some costs, such as interest, maintenance, and repair, have fixed cost while maintenance and repair are considered variable costs.

\subsubsection{Fixed cost}

Fixed costs considered in the study are depreciation, local taxes, insurance, plant overhead, administration, interest. Direct labor is estimated to be 150 employees including engineers, technician, accountants, HR representatives ...etc. Expected average salaries will be around 1800 US \$ /month for each employee, so total annual salaries will be nearly 3.3 million US \$.Maintenance and insurance will be $2 \%$ of total direct material or purchased equipment cost so the both of maintenance and insurance cost will be 280 thousands $\$$ per year.

\subsubsection{Variable Cost}

Variable costs considered in study are: raw material, utilities, and chemicals. Raw materials such as methanol and biodiesel prices was obtained from ICIS weekly pricing report while price of potassium hydroxide price obtained from website [4] and price of used cooking obtained by local survey. The prices of feedstock waste cooking oil is 400 US \$/ton according to the prices from local market.

Utilities include steam, utility water, process water, cooling water, electricity ...etc. The prices of utilities are obtained from other projects executed in Egypt especially petrochemical projects. In this project only steam, utility water, process water and electricity will be utilized during process. The prices of utilities are detailed in below table 7 . Chemicals used in biodiesel production are dedicated for treatment of cooling water. Chemicals used such as sodium bisulfate for removing free chlorine and organic compound, ion exchange resin (strong acid ) for removing water scaling caustic soda or sulphuric acid for PH neutralization, corrosion inhibitors. The expected price of these chemicals estimated as three million \$ per year. 
Table 6: Expected feedstocks \& product prices till 2038

\begin{tabular}{|c|c|c|c|c|c|c|c|c|c|c|c|c|c|c|c|c|c|c|c|c|}
\hline & त्रे & ث્તે & )ิ艹 & ฮี่ & c్đ: & ड़ें & हैं & 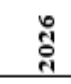 & 월 & 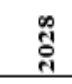 & त्̀े & 芦 & है & है & 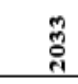 & 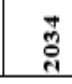 & 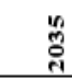 & 足 & 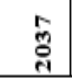 & 恖 \\
\hline \multicolumn{21}{|l|}{ Prices } \\
\hline \multicolumn{21}{|c|}{ Feedstock (USD per ton) } \\
\hline Methanol & 441 & 454 & 467 & 481 & 496 & 511 & 526 & 542 & 558 & 575 & 592 & 610 & 628 & 647 & 666 & 686 & 707 & 728 & 750 & 772 \\
\hline $\mathrm{KOH}$ & 1,043 & 1,075 & 1,107 & 1,140 & 1,174 & 1,210 & 1,246 & 1,283 & 1,322 & 1,361 & 1,402 & 1,444 & 1,488 & 1,532 & 1,578 & 1,626 & 1,674 & 1,724 & 1,776 & 1,830 \\
\hline Used Oil & 464 & 478 & 492 & 507 & 522 & 538 & 554 & 570 & 587 & 605 & 623 & 642 & 661 & 681 & 701 & 722 & 744 & 766 & 789 & 813 \\
\hline \multicolumn{21}{|c|}{ Products (USD per ton) } \\
\hline Biodiesel & 777 & 800 & 824 & 849 & 874 & 900 & 927 & 955 & 984 & 1,013 & 1,044 & 1,075 & 1,107 & 1,141 & 1,175 & 1,210 & 1,246 & 1,284 & 1,322 & 1,362 \\
\hline
\end{tabular}

Table 7: Expected utilities prices till 2038

\begin{tabular}{|c|c|c|c|c|c|c|c|c|c|c|c|c|c|c|c|c|c|c|c|c|}
\hline & ڤ્సి & : & ड્ّ & : & त्व̃ & ड્ّ & :ัّ & :ัّ & 今ે & :ึّ & त्षे & : & 颉 & 象 & 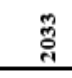 & 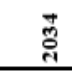 & 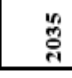 & ֻั. & 突 & 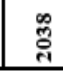 \\
\hline \multicolumn{21}{|l|}{ Utilities } \\
\hline Water $\left(\mathrm{USD} / \mathrm{m}^{3}\right)$ & 0.6 & 1 & 1 & 1 & 1 & 1 & 1 & 1 & 1 & 1 & 1 & 1 & 1 & 1 & 1 & 1 & 1 & 1 & 1 & 1 \\
\hline $\begin{array}{l}\text { Steam } \\
\text { Consumptions } \\
\text { USD/ton) }\end{array}$ & 10.4 & 11 & 11 & 11 & 12 & 12 & 12 & 13 & 13 & 14 & 14 & 14 & 15 & 15 & 16 & 16 & 17 & 17 & 9 & 9 \\
\hline $\begin{array}{l}\text { Electricity } \\
\text { Consumption } \\
\text { (USD/MWH) }\end{array}$ & 87 & 90 & 92 & 95 & 98 & 101 & 104 & 107 & 110 & 113 & 117 & 120 & 124 & 128 & 132 & 135 & 140 & 144 & 75 & 77 \\
\hline $\begin{array}{l}\text { Land Lease } \\
\text { (USD } / \mathrm{m}^{3} \text { ) }\end{array}$ & 0 & 0 & 0 & 0 & 0 & 0 & 0 & 0 & 0 & 0 & 0 & 0 & 0 & 0 & 0 & 0 & 0 & 0 & 0 & 0 \\
\hline $\begin{array}{l}\text { Labour } \\
\text { (USD/Worker) }\end{array}$ & 20,86 & 21,49 & 22,138 & 22,802 & 23,486 & 24,190 & 24,916 & 25,664 & 26,434 & 27,227 & 28,043 & 28,885 & 29,751 & 30,644 & 31,563 & 32,510 & 33,49 & 34,49 & 35,53 & 36,59 \\
\hline
\end{tabular}




\begin{tabular}{|c|c|c|c|c|c|c|c|c|c|c|c|c|c|c|c|c|c|c|c|c|}
\hline & छे & : & خ્તે & ণ્̀ે & 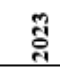 & ड़̀ं & 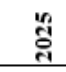 & 율 & 今ે̀ & 商 & त्रे & 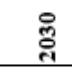 & क्षे & 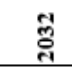 & 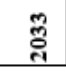 & 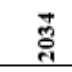 & 赵 & 芯 & हैं & 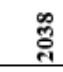 \\
\hline \multicolumn{21}{|c|}{ Fixed Costs (M\$) } \\
\hline $\begin{array}{l}\text { Total Fixed } \\
\text { Costs } \\
\text { MMUSD }\end{array}$ & 3 & 3 & 4 & 4 & 4 & 5 & 5 & 5 & 6 & 6 & 7 & 7 & 8 & 9 & 9 & 10 & 11 & 12 & 13 & 14 \\
\hline \multicolumn{21}{|c|}{ Variable Costs (M\$) } \\
\hline \multicolumn{21}{|c|}{ 1. Feedstock Cost } \\
\hline $\begin{array}{l}\text { Methanol } \\
\text { Cost }\end{array}$ & 9 & 11 & 13 & 13 & 13 & 14 & 14 & 15 & 15 & 15 & 16 & 16 & 17 & 17 & 18 & 18 & 19 & 20 & 20 & 21 \\
\hline $\mathrm{KOH}$ Cost & 1 & 1 & 1 & 1 & 1 & 1 & 1 & 1 & 1 & 1 & 1 & 1 & 1 & 1 & 1 & 1 & 1 & 2 & 2 & 2 \\
\hline Oil Cost & 37 & 43 & 50 & 51 & 53 & 54 & 56 & 58 & 59 & 61 & 63 & 65 & 67 & 69 & 71 & 73 & 75 & 77 & 80 & 82 \\
\hline $\begin{array}{l}\text { Total } \\
\text { Feedstocks } \\
\text { Cost }\end{array}$ & 48 & 55 & 63 & 65 & 67 & 69 & 71 & 73 & 75 & 78 & 80 & 82 & 85 & 87 & 90 & 93 & 96 & 98 & 101 & 104 \\
\hline \multicolumn{21}{|l|}{ 2. Utilities } \\
\hline $\begin{array}{l}\text { Water } \\
\text { Cost }\end{array}$ & 0.7 & 1 & 1 & 1 & 1 & 1 & 1 & 1 & 1 & 1 & 1 & 1 & 1 & 1 & 1 & 1 & 1 & 1 & 1 & 1 \\
\hline $\begin{array}{l}\text { Electricity } \\
\text { Cost }\end{array}$ & 1 & 1 & 1 & 1 & 1 & 2 & 2 & 2 & 2 & 2 & 2 & 2 & 2 & 2 & 2 & 2 & 2 & 2 & 2 & 2 \\
\hline $\begin{array}{l}\text { Steam } \\
\text { Cost }\end{array}$ & 3 & 3 & 3 & 3 & 4 & 4 & 4 & 4 & 4 & 4 & 4 & 4 & 4 & 5 & 5 & 5 & 5 & 5 & 5 & 5 \\
\hline $\begin{array}{l}\text { Total } \\
\text { Utility } \\
\text { Cost } \\
\end{array}$ & 4 & 5 & 5 & 5 & 6 & 6 & 6 & 6 & 6 & 7 & 7 & 7 & 7 & 7 & 8 & 8 & 8 & 8 & 9 & 9 \\
\hline & $\stackrel{2}{8}$ & 윰 & त्ञ & cid & 象 & : & di & : & 今̀ & 艧 & त्रे & : & हैं & ç & \%ั. & $\stackrel{B}{M}_{i}^{*}$ & $\overbrace{\substack{n \\
0}}^{n}$ & : & ồ & 悉 \\
\hline $\begin{array}{l}\text { 3.Chemicals } \\
\text { and } \\
\text { Catalyst }\end{array}$ & 3 & 3 & 3 & 3 & 3 & 3 & 4 & 4 & 4 & 4 & 4 & 4 & 4 & 4 & 5 & 5 & 5 & 5 & 5 & 5 \\
\hline $\begin{array}{l}\text { Total } \\
\text { Variable } \\
\text { Costs } \\
\text { MMUSD }\end{array}$ & 55 & 63 & 72 & 74 & 76 & 78 & 81 & 83 & 86 & 88 & 91 & 94 & 96 & 99 & 102 & 105 & 108 & 112 & 115 & 118 \\
\hline $\begin{array}{l}\text { Total Opex } \\
\text { Cost } \\
\text { MMUSD }\end{array}$ & 58 & 66 & 75 & 78 & 80 & 83 & 86 & 89 & 91 & 94 & 98 & 101 & 104 & 108 & 111 & 115 & 119 & 123 & 128 & 132 \\
\hline
\end{tabular}


Table 9: Revenue of biodiesel project till 2038

\begin{tabular}{|c|c|c|c|c|c|c|c|c|c|c|c|c|c|c|c|c|c|c|c|c|}
\hline & हैं & : & 혹 & 렴 & 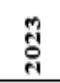 & 突 & 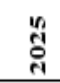 & 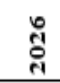 & 今̀ & 芯 & त्रे & 芦 & $\overline{\mathrm{c}}$ & $\overbrace{\mathrm{A}}^{\mathrm{A}}$ & 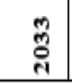 & $\overbrace{\vec{A}}^{\vec{m}}$ & 常 & 茫 & 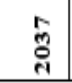 & 赵 \\
\hline & $80 \%$ & $90 \%$ & \multicolumn{18}{|c|}{$100 \%$} \\
\hline $\begin{array}{l}\text { Biodiesel } \\
\text { (\$/Ton) }\end{array}$ & 777 & 800 & 824 & 849 & 874 & 900 & 927 & 955 & 984 & 1,013 & 1,044 & 1,075 & 1,107 & 1,141 & 1,175 & 1,210 & 1,246 & 1,284 & 1,322 & 1,362 \\
\hline $\begin{array}{l}\text { Biodiesel } \\
\text { Production } \\
\text { (thousand } \\
\text { Tons per } \\
\text { year) }\end{array}$ & 80,000 & 90,000 & \multicolumn{18}{|c|}{100,000} \\
\hline $\begin{array}{l}\text { Product } \\
\text { Income } \\
\text { (MMUSD) }\end{array}$ & 50 & 65 & 82 & 85 & 87 & 90 & 93 & 96 & 98 & 101 & 104 & 108 & 111 & 114 & 117 & 121 & 125 & 128 & 132 & 136 \\
\hline \begin{tabular}{l}
\multicolumn{1}{c}{ Total } \\
Revenues \\
(MMUSD)
\end{tabular} & 50 & 65 & 82 & 85 & 87 & 90 & 93 & 96 & 98 & 101 & 104 & 108 & 111 & 114 & 117 & 121 & 125 & 128 & 132 & 136 \\
\hline
\end{tabular}

Table 10: Income statement

\begin{tabular}{|c|c|c|c|c|c|c|c|c|c|c|c|c|c|c|c|c|c|c|c|c|}
\hline & : & : & ฮี & ક્ત & c్đ & त्̀ं & ç & 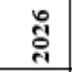 & ç્đ & 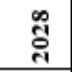 & त्रे & 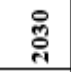 & : & ठิ & 商 & 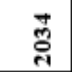 & 品 & 品 & 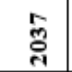 & 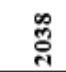 \\
\hline $\begin{array}{l}\text { Total } \\
\text { Revenues } \\
\text { MMS }\end{array}$ & 50 & 65 & 82 & 85 & 87 & 90 & 93 & 96 & 98 & 101 & 104 & 108 & 111 & 114 & 117 & 121 & 125 & 128 & 132 & 136 \\
\hline $\begin{array}{l}\text { Total } \\
\text { Opex } \\
\text { MMUSD }\end{array}$ & 58 & 67 & 76 & 78 & 81 & 84 & 86 & 89 & 92 & 95 & 98 & 102 & 105 & 109 & 112 & 116 & 120 & 124 & 129 & 133 \\
\hline $\begin{array}{l}\text { True } \\
\text { Tax }\end{array}$ & - & - & 1.54 & 1.55 & 1.56 & 1.56 & 1.56 & 1.56 & 1.54 & 1.51 & 1.48 & 1.44 & 1.40 & 1.34 & 1.28 & 1.20 & 1.11 & 1.02 & 0.90 & 0.77 \\
\hline $\begin{array}{l}\text { Income } \\
\text { After } \\
\text { Tax } \\
\end{array}$ & - & - & 5.03 & 5.07 & 5.10 & 5.12 & 5.14 & 5.16 & 5.12 & 5.06 & 4.98 & 4.88 & 4.76 & 4.61 & 4.43 & 4.22 & 3.98 & 3.70 & 3.39 & 3.02 \\
\hline IRR & 28.1 & & & & & & & & & & & & & & & & & & & \\
\hline $\begin{array}{l}\text { Pay Back } \\
\text { Period }\end{array}$ & $7.6 \mathrm{Y}$ & ears & & & & & & & & & & & & & & & & & & \\
\hline NPV & $16 \mathrm{M}$ & [USS & & & & & & & & & & & & & & & & & & \\
\hline
\end{tabular}




\section{Discussion}

The comparison between total revenue and total OPEX is shown in fig 5 demonstrates that at first two years the OPEX will be higher than total revenue till 2020 the total revenue become higher and the project begin to be more profitable. The total revenue will be higher till end of the project. The net income or net profit is one of the financial statements shows the project's revenues and expenses during a particular period. The net come in simple way is the difference between total revenue and expenses that include total OPEX, taxes. Fig 6 shows that biodiesel production cost is lower than petro-diesel price enhancing that biodiesel is more economical and profitable than fossil fuel. Fig 7 shows the variation of net income during all 20 years of the project; the income in 2019 and 2020 is zero as project will not achieve any profit during this years. Starting from 2021 the project will achieve good income near 5.03 million US \$ and verified in next year reaching 3 million $\$$ at the end of project. Table 11 shows an estimation of production one litre of biodiesel, the current price of biodiesel $3.77 \$$ /gallon or 1 $\$$ /litre, for petro-diesel is 2.56 /gallon or 0.677 \$/litre while production of one litre of biodiesel is $0.515 \$$, this enhancing the economical of changing to biodiesel production from waste cooking oil.

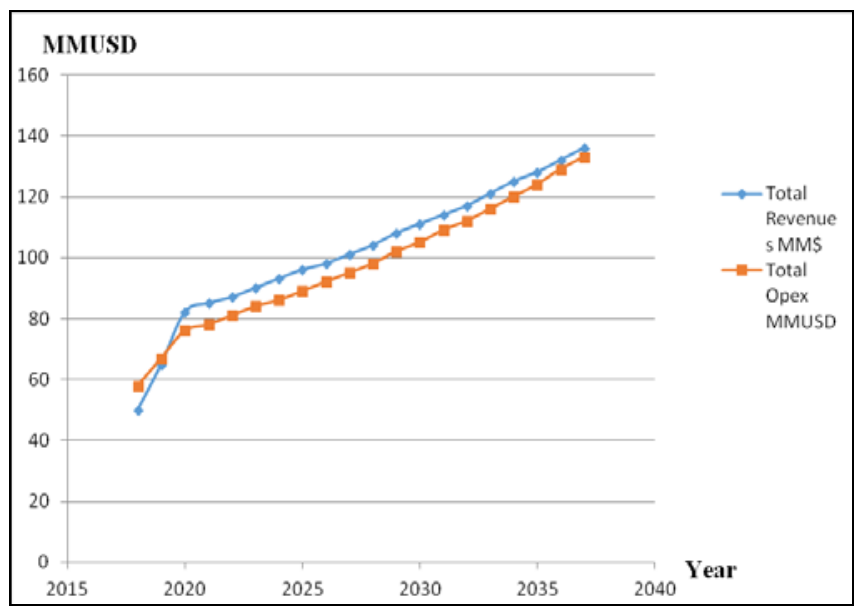

Fig 6: Total revenue versus Total OPEX

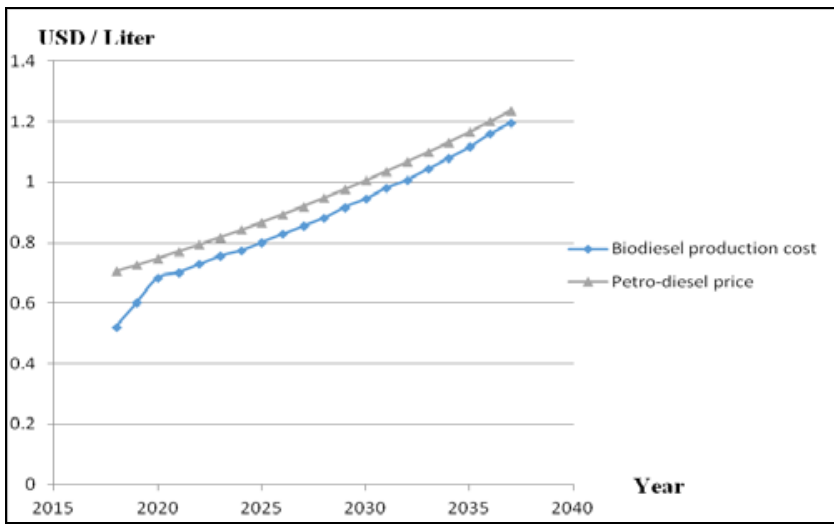

Fig 7: Biodiesel production cost \& Petro-diesel price
Table 11: An Estimation for production of 1 litre biodiesel

\begin{tabular}{|l|c|}
\hline Item & Price \\
\hline Methanol cost (\$/litre biodiesel) & 0.072 \\
\hline KOH cost (\$/litre biodiesel) & 0.02 \\
\hline Waste cooking oil cost (\$/litre biodiesel) & 0.333 \\
\hline Utilities cost (\$/litre biodiesel) & 0.036 \\
\hline Other chemicals costs (\$/litre biodiesel) & 0.027 \\
\hline $\begin{array}{l}\text { Fixed cost (maintenance, salaries and } \\
\text { insurance) (\$/litre biodiesel) }\end{array}$ & 0.027 \\
\hline Biodiesel production cost (\$/litre) & 0.515 \\
\hline $\begin{array}{l}\text { Biodiesel production cost (\$/gallon) } \\
\text { Current market price biodiesel (\$/gallon) [5] }\end{array}$ & 3.77 \\
\hline $\begin{array}{l}\text { Current market price petro-diesel in USA } \\
\text { \$/gallon) [6] }\end{array}$ & 2.561 \\
\hline $\begin{array}{l}\text { Current market price petro-diesel in Egypt } \\
\text { \$/gallon) }\end{array}$ & 0.871 \\
\hline
\end{tabular}

\section{Conclusion}

Biodiesel project is very profitable, the economic indicators for the project is very high and execution of such a project will strength the national economic in addition provide about 150 direct job opportunity and 1000 non-direct job. The total CAPEX needed to build up the biodiesel plant is 4.121 million $\$$ as the purchased equipment cost is 1.3 million $\$$ while other CAPEX is 2.8 million $\$$.

The total OPEX will be 58 million US \$ in 2019 reaches up to 132 million US \$ in 2038 while the revenue in 2019 will be 50 million US $\$$ increasing gradually every year reaching to 136 million US \$ in 2038.

The net income or profit is the difference between the revenue and the total OPEX, there will be a variation in net income during all 20 years of the project. The project will not achieve any profit during the first two years in contrast while from 2021 the project will achieve a net income about 5.03 million US \$ verifying in the next years up and down achieving a total net income during the life cycle of project about 72.5 million US \$. Cost analysis concluded that the current price of biodiesel $3.77 \$$ /gallon or 1 \$/litre and for petro-diesel is 2.56 \$/gallon or $0.677 \$$ /litre while the cost of production one litre of biodiesel is $0.515 \$$. The production of one litre of biodiesel costs 0.515 US \$ in 2018 increasing up to $1.1 \mathrm{US} \$$ at the end of project life cycle while one litre of petro-diesel international price in 2018 is 0.7 US \$ expecting to increase up to 1.24 US \$ in 2038.

Accelerate in Changing to utilization of biodiesel from waste cooking oil will have a great profit and positive effect on environment, vehicle engines, independency on crude oil, investment and economy as it will create jobs and save foreign currency especially that Egypt consumption of petrodiesel is very high. Egypt consumption of petro-diesel is 13.5 
million ton in 2014, Egypt imports $25 \%$ of diesel and 75\% from local market, and there is no accurate information about waste cooking oil quantity in Egypt. Cost of petro-diesel production is 1 \$ while cost production of one litre biodiesel is $0.515 \$$, so building up biodiesel plants will no doubt decrease dependences on petro-diesel, save hard currency of importing petro-diesel [7].

\section{References}

[1] Ertan Alptekin ,Mustafa Canakci Huseyin Sanli ,2011, Methyl ester production from chicken fat with high FFA , world renewable energy congress, 8-13.

[2] Surachai Jansria, Sukritthira B. Ratanawilaib, Michael L. Allenc, Gumpon Prateepchaikulc ,2011,Kinetics of methyl ester production from mixed crude palm oil by using acidalkali catalyst, Fuel Processing Technology Volume 92, Issue 8, Pages 1543-1548.

[3] INFORMS website, May 2016: https://www.extension.org/pages/26611/biodiesel-cloudpoint-and-cold-weather-issues\#.VAv4MPmSxqU (Last accessed on December 27, 2017)

[4] INFORMS website, Aug 2016: http://www.afdc.energy.gov/uploads/publication/alternativ e_fuel_price_report_april_2015.pdf (Last accessed on December 27, 2017)

[5] INFORMS website, Aug 2016: http://www.eia.gov/dnav/pet/pet_pri_gnd_dcus_nus_w.ht m (Last accessed on December 27, 2017)

[6] INFORMS website, Dec 2016:

http://www.dailynewsegypt.com/2014/07/06/fuel- consumption-rates-stabilise-domestic-marketimplementation-price-increases/ (Last accessed on December 27, 2017)

[7] INFORMS website ,Dec 2016:

http://www.dailynewsegypt.com/2014/07/06/fuelconsumption-rates-stabilise-domestic-marketimplementation-price-increases/ (Last accessed on December 27, 2017) 